ARTICLE

\title{
Changes in rates of adverse pregnancy outcomes during the COVID-19 pandemic: a cross-sectional study in the United States, 2019-2020
}

Regina M. Simeone $\mathbb{I D}^{1 凶}$, Karrie F. Downing $\mathbb{D}^{1}{ }^{1}$, Bailey Wallace $\mathbb{D}^{1}{ }^{1}$, Romeo R. Galang ${ }^{1}{ }^{1}$, Carla L. DeSisto (iD) ${ }^{1}$, Van T. Tong ${ }^{1}$, Lauren B. Zapata (iD ${ }^{1,2}$, Jean Y. Ko (iD) ${ }^{1,2}$ and Sascha R. Ellington (iD)

This is a U.S. government work and not under copyright protection in the U.S.; foreign copyright protection may apply 2022

\begin{abstract}
OBJECTIVE: Our objective was to assess differences in pregnancy outcomes during the COVID-19 pandemic compared to the previous year.

STUDY DESIGN: In a cross-sectional study of delivery hospitalizations in the Premier Healthcare Database Special COVID-19 Release, we assessed differences in selected maternal and pregnancy outcomes occurring April-December in 2019 and 2020 in the United States.

RESULT: Among 663,620 deliveries occurring in 2019 and 614,093 deliveries occurring in 2020, we observed an increase in inhospital maternal death from 2019 to 2020, which was no longer statistically significant after excluding deliveries with a COVID-19 diagnosis. Intensive care unit admission and preterm birth decreased from 2019 to 2020 . There was no difference in the prevalence of most other outcomes examined.

CONCLUSION: The full impact of the COVID-19 pandemic on maternal and pregnancy outcomes remains to be understood. Most outcomes investigated experienced minimal change from 2019 to 2020.
\end{abstract}

Journal of Perinatology (2022) 42:617-623; https://doi.org/10.1038/s41372-022-01327-3

\section{INTRODUCTION}

The immense scale of the coronavirus disease 2019 (COVID-19) pandemic has resulted in substantial changes to healthcare delivery throughout the United States, including reductions in the use of preventive care and other health services [1, 2]. The delivery of obstetric care, including prenatal and intrapartum services, has also been impacted [3-6]. Stay-at-home orders that were declared across the country in 2020 and intended to mitigate the spread of the virus required abrupt changes to individuals' work, social, and physical activity habits. Additionally, social isolation and increased caregiving responsibilities have been identified as potential negative influences impacting health [7-10]. Changes in healthcare policies, utilization, and access, as well as increased time at home, stress, and mental health concerns could impact the health and healthcare of pregnant people.

Compared to pregnant persons without severe acute respiratory syndrome coronavirus 2 (SARS-CoV-2) infection, the virus that causes COVID-19, pregnant persons with SARS-CoV-2 are at increased risk of preterm birth and may be at increased risk of other adverse pregnancy outcomes, including stillbirth [11-17]. Further, pregnant and recently pregnant persons with SARS-CoV-2 infection are at increased risk of severe COVID-19 illness compared to non-pregnant people $[13,18]$. Less is known about whether non-COVID-19 related maternal outcomes are different than before the pandemic [19-21]. Early in the pandemic, reports from outside of the United States detailed reductions in preterm birth and low birth weight infants after the implementation of COVID-19 mitigation measures, including stay-at-home orders [22-26]. A recent study examining commercial and Medicaid U.S. claims observed a slight reduction in preterm birth and no change in stillbirth from 2019 to 2020 [27]. Another U.S. cohort study of women delivering during the COVID-19 pandemic found that the occurrence of selected adverse pregnancy outcomes, including preterm birth and stillbirth, did not change compared to the past year [28]. However, results of other U.S. studies assessing the impact of COVID-19 mitigation measures on preterm birth have been mixed [29-34]. Pandemic-related changes in adverse maternal and pregnancy outcomes may vary based on country-level resources and within-state differences. For example, a recent systematic review observed reductions in preterm birth only in high-income countries, whereas significant increases in maternal and fetal mortality were more often observed in low-income and middle-income countries [19, 29-33].

Within the context of the COVID-19 pandemic, many hospitals implemented infection control measures to help protect hospital staff, patients, and caregivers from contracting COVID-19 $[5,35,36]$. Measures included SARS-CoV-2 screening for women upon hospital admission, restricting the number of support persons and visitors for laboring women, and policies for masks and personal protective equipment in healthcare facilities. Additionally, the American College of Obstetricians and

\footnotetext{
${ }^{1}$ COVID-19 Response, Centers for Disease Control and Prevention, Atlanta, GA, USA. ${ }^{2}$ United States Public Health Service, Commissioned Corps, Rockville, MD, USA. email: eocevent397@cdc.gov
}

Received: 28 October 2021 Revised: 21 December 2021 Accepted: 25 January 2022

Published online: 15 February 2022 
Gynecologists (ACOG) provided guidance clarifying that SARSCoV-2 infection is not an indication for cesarean delivery and that labor induction and cesarean delivery should be based on obstetric indications and not COVID-19 status [5]. ACOG additionally recommended expedited discharge of mother-infant dyads when appropriate to help prevent SARS-CoV-2 infection during the delivery hospitalization [5].

The collective impact of the COVID-19 pandemic, including mitigation measures, on the delivery of obstetric care is not well understood. Previous U.S. studies have been limited in the outcomes they could observe or assessed outcomes in single hospital centers. Thus, using data from a large multi-state U.S. hospital-based administrative database, we compared the prevalence of selected maternal, delivery, and pregnancy outcomes occurring in 2020 to those in 2019.

\section{MATERIALS AND METHODS \\ Data}

We performed a cohort analysis of delivery hospitalizations using the Premier Healthcare Database Special COVID-19 Release (https://www. premierinc.com/; PHD-SR, release date 8/15/2021), which includes data from 2019 onward. The PHD-SR is a U.S. hospital-based, service-level, allpayor database. It represents approximately $20 \%$ of all U.S. inpatient admissions, regardless of payor type, from geographically diverse nonprofit, non-governmental, community, and teaching hospitals and health systems from rural and urban areas [37]. We included hospitals that contributed data for at least one maternal delivery discharge during AprilDecember in both 2019 and 2020 and restricted the sample to the same hospitals each year.

Delivery hospitalizations were identified among women aged 15-45 years using International Classification of Diseases, 10th revision, Clinical Modification (ICD-10-CM) diagnostic and procedure codes, as well as diagnostic related group delivery codes, for singleton live birth or stillbirth deliveries on the patient diagnosis and procedure tables (Appendix 1). Deliveries with codes for multiple births were excluded due to the association between multiple gestations and preterm delivery. Deliveries were limited to those discharged from April $1^{\text {st }}$-December 31st in 2020 or in 2019. Gestational age at delivery was assessed using Z3A.xx codes; pregnancies that ended prior to 20 weeks gestation or after 42 weeks gestation were excluded. If a woman had more than one delivery hospitalization coded as occurring in the same year, with hospitalizations greater than 30 days apart or with a difference in estimated gestational age that changed the classification of preterm vs. term birth, she was excluded from the analysis ( $n=239$ women/485 encounters in 2019 and 183 women/375 encounters in 2020). If a woman had more than one delivery hospitalization coded the same year, but with hospitalizations less than or equal to 30 days apart and with the same preterm/term categorization, the first delivery hospitalization encounter was included, and subsequent encounters were excluded ( $n=1114$ women contributing 1127 excluded encounters in 2019 and 1060 women contributing 1070 encounters in 2020).

Maternal demographic characteristics included maternal age, race and ethnicity, marital status, and primary payor. Maternal race and ethnicity are collected separately in the PHD-SR and were combined for this analysis. Women with records indicating Hispanic ethnicity were considered Hispanic, regardless of recorded or missing race. Women missing Hispanic ethnicity or categorized as non-Hispanic were classified as non-Hispanic and assigned their recorded race. Race was missing in 3\% of observations. We additionally considered U.S. Census division location and urban/rural status as hospital characteristics.

We identified the following maternal comorbidities, which are associated with adverse pregnancy outcomes and increased risk of cesarean delivery: hypertensive disorders (chronic hypertension, gestational hypertension, chronic hypertension with superimposed pre-eclampsia, pre-eclampsia, Hemolysis, elevated liver enzymes, low platelet count (HELLP) syndrome, and eclampsia), diabetes (Type 1, Type 2, gestational, other/unspecified), and obesity (Appendix 2).

\section{Outcomes}

We identified selected adverse maternal and pregnancy outcomes if an ICD-10-CM code was present on the delivery hospitalization record. Our primary outcomes were maternal intensive care unit (ICU) admission, maternal in-hospital death, preterm birth, and stillbirth. Maternal ICU admission was identified in the hospital chargemaster records, and inhospital death was identified using the maternal discharge status code (Appendix 3). Pregnancies with a gestational age of less than 37 weeks were considered preterm; pregnancies with a gestational age of 37 to 42 weeks were considered term.

Secondary outcomes included mode of delivery (vaginal or cesarean), and cesarean delivery characteristics. Among cesarean deliveries, we identified those with premature rupture of membranes (PROM), prolonged labor, attempted assisted vaginal delivery by forceps or vacuum, or augmentation or induction of labor (Appendix 3). These classifications were not mutually exclusive. We further identified cesarean deliveries considered to be at low risk for cesarean delivery using the Society for Maternal-Fetal Medicine (SMFM) updated definition [38]. Those excluded from the low-risk category (hereafter referred to as high-risk) were further categorized into the following non-mutually exclusive groups: maternal factors (e.g., HIV disease, eclampsia, congenital cardiovascular disorders of mother), preterm birth, stillborn, malpresentation (e.g., breech presentation), fetal factors (e.g., hydrocephalic fetus), uterine/placental factors (e.g., placenta previa, previous cesarean delivery), and conduct of labor (e.g., failed forceps or vacuum extraction) (Appendix 3).

Finally, we examined maternal inpatient readmission to the same hospital within 30 days. We additionally examined the overall distribution of delivery length of stay (vaginal: $<1$ day, 1 day, 2 days, $\geq 3$ days; cesarean: $<1$ day, 1-2 days, 3 days, 4 days, $\geq 5$ days), stratified by mode of delivery.

\section{Statistical analysis}

We report the distribution of maternal and hospital characteristics and adverse maternal and pregnancy outcomes in 2020 compared to 2019. Poisson regression models, with robust standard errors, were used to estimate unadjusted and adjusted prevalence ratios (aPRs) for adverse maternal and pregnancy outcomes comparing those occurring in 2020 to those occurring in 2019 [39]. For the assessment of delivery length of stay, we assessed the overall differences in the distribution of categorical length of stay in 2019 and 2020 using Pearson chi-square tests. We also ran separate Poisson regression models, comparing each category of length of stay (defined above) to a reference category (vaginal deliveries: two days; cesarean deliveries: three days). Data recorded in unique hospitals may be correlated; additionally, 20,458 women delivered in both 2019 and 2020. We accounted for correlations within individuals, nested within hospitals, using an exchangeable correlation matrix. We, a priori, considered the following covariates: maternal age, census division, primary payor, and maternal comorbidities. Models were adjusted for different subsets of covariates, depending on the outcome. A two-sided $p$-value $<0.05$ was considered statistically significant.

As a sensitivity analysis, we excluded women with a COVID-19 diagnosis (ICD-10-CM code U07.1 from April 2020-December 2020 or B97.29 during April 2020) [40] at the delivery hospitalization to assess if the direct effect of SARS-CoV-2 infection during pregnancy was the primary contributor to differences in adverse outcomes. As a second sensitivity analysis, we stratified our analyses by quarter (Quarter 2: April-June; Quarter 3: July-September; Quarter 4: October-December) to assess whether changes in selected outcomes differed over the course of the pandemic compared to the same time-period the previous year. Because analyses of large datasets can result in statistically significant findings that may not be meaningful, we a priori chose to report results of cesarean delivery characteristics if they had an aPR indicating at least 5\% change in 2020 compared to 2019. All analyses were performed in SAS 9.4 (SAS Institute, Cary, NC). Analytic code is available upon request to the first author.

This activity was reviewed by CDC and was conducted consistent with applicable federal law and CDC policy (See e.g., 45C.F.R. part 46, 21C.F.R. part 56; 42 U.S.C. $\$ 241$ (d); 5 U.S.C. §552a; 44 U.S.C. $\$ 3501$ et seq.).

\section{RESULTS}

There were 664 hospitals that reported at least one delivery discharged during April through December in both 2019 and 2020. We identified 663,620 deliveries occurring during April through December of 2019 and 614,093 deliveries occurring during April through December of 2020 from these hospitals (Table 1, Appendix 4). Maternal demographic characteristics were similar in 2019 and 2020, with slightly more deliveries in 2020 occurring among women of Hispanic ethnicity, and slightly fewer deliveries in 2020 occurring among married women. Hospital characteristics were also 
Table 1. Maternal and hospital characteristics of deliveries occurring from April through December of 2019 and 2020, Premier Healthcare Database Special COVID-19 Release, United States.

\begin{tabular}{|c|c|c|c|c|}
\hline \multirow[b]{2}{*}{ Characteristics } & \multicolumn{2}{|l|}{2019} & \multicolumn{2}{|l|}{2020} \\
\hline & $n$ & $\%$ & $n$ & $\%$ \\
\hline Total deliveries & 663,620 & & 614,093 & \\
\hline Maternal age (mean, SD) & 29 & 5.8 & 29 & 5.8 \\
\hline \multicolumn{5}{|l|}{ Maternal race and ethnicity ${ }^{a}$} \\
\hline Non-Hispanic White & 358,484 & 54.0 & 329,907 & 53.7 \\
\hline Hispanic & 113,322 & 17.1 & 110,212 & 17.9 \\
\hline Non-Hispanic Black & 92,388 & 13.9 & 88,813 & 14.5 \\
\hline Non-Hispanic Asian & 29,777 & 4.5 & 26,706 & 4.3 \\
\hline Non-Hispanic Other & 50,951 & 7.7 & 40,592 & 6.6 \\
\hline Missing & 18,698 & 2.8 & 17,863 & 2.9 \\
\hline \multicolumn{5}{|l|}{ Marital status } \\
\hline Married & 320,420 & 48.3 & 289,520 & 47.2 \\
\hline Single & 266,728 & 40.2 & 251,634 & 41.0 \\
\hline Other & 70,424 & 10.6 & 65,876 & 10.7 \\
\hline Unknown & 6048 & 0.9 & 7063 & 1.2 \\
\hline \multicolumn{5}{|l|}{ Primary payor ${ }^{\mathrm{b}}$} \\
\hline Private & 333,695 & 50.3 & 308,858 & 50.3 \\
\hline Medicaid & 286,231 & 43.1 & 266,696 & 43.4 \\
\hline Self-pay & 14,866 & 2.2 & 11,373 & 1.9 \\
\hline Other & 28,828 & 4.3 & 27,166 & 4.4 \\
\hline \multicolumn{5}{|l|}{ Census division $^{c}$} \\
\hline East North Central & 104,432 & 15.7 & 99,504 & 16.2 \\
\hline East South Central & 49,242 & 7.4 & 46,184 & 7.5 \\
\hline Middle Atlantic & 85,016 & 12.8 & 75,175 & 12.2 \\
\hline Mountain & 47,630 & 7.2 & 44,612 & 7.3 \\
\hline New England & 12,040 & 1.8 & 12,186 & 2.0 \\
\hline Pacific & 80,993 & 12.2 & 62,148 & 10.1 \\
\hline South Atlantic & 165,558 & 25.0 & 162,799 & 26.5 \\
\hline West North Central & 40,820 & 6.2 & 37,538 & 6.1 \\
\hline West South Central & 77,889 & 11.7 & 73,947 & 12.0 \\
\hline \multicolumn{5}{|l|}{ Hospital location ${ }^{d}$} \\
\hline Urban & 581,946 & 87.7 & 538,636 & 87.7 \\
\hline Rural & 81,674 & 12.3 & 75,457 & 12.3 \\
\hline \multicolumn{5}{|l|}{ Maternal comorbidities } \\
\hline Hypertensive disorders of pregnancy & 99,324 & 15.0 & 101,013 & 16.5 \\
\hline Diabetes ( $\mathrm{T} 1 / \mathrm{T} 2$ gestational other) & 63,989 & 9.6 & 68,432 & 11.1 \\
\hline Type 1 or type 2 diabetes & 7419 & 1.1 & 7630 & 1.2 \\
\hline Gestational diabetes & 55,738 & 8.4 & 59,852 & 9.8 \\
\hline Obesity & 84,417 & 12.7 & 91,670 & 14.9 \\
\hline
\end{tabular}

Abbreviations: SD Standard deviation, T1/T2 Type 1 or Type 2 .

aMaternal race and ethnicity collected separately and combined; Women with records indicating Hispanic ethnicity were considered Hispanic, regardless of recorded or missing race. Women missing Hispanic ethnicity or categorized as non-Hispanic were classified as non-Hispanic and assigned their recorded race.

bPrivate payor includes managed care and commercial indemnity.

'East North Central: Indiana, Illinois, Michigan, Ohio, Wisconsin; East South Central: Alabama, Kentucky, Mississippi, Tennessee; Middle Atlantic: New Jersey, New York, Pennsylvania; Mountain: Arizona, Colorado, Idaho, New Mexico, Montana, Utah, Nevada, Wyoming; New England: Connecticut, Maine, Massachusetts, New Hampshire, Rhode Island, Vermont; Pacific: Alaska, California, Hawaii, Oregon, Washington; South Atlantic: Delaware, District of Columbia, Florida, Georgia, Maryland, North Carolina, South Carolina, Virginia, West Virginia; West North Central: lowa, Kansas, Minnesota, Missouri, Nebraska, North Dakota, South Dakota; West South Central: Arkansas, Louisiana, Oklahoma, Texas.

${ }^{\mathrm{d}}$ The U.S. Census defines an urban area as a territory whose core census block groups or blocks have a population density of at least 1000 people per square mile, and surrounding census blocks have an overall density of at least 500 people per square mile. Rural areas are considered territory outside the definition of urban.

eIncludes chronic hypertension, gestational hypertension, chronic hypertension with superimposed pre-eclampsia, pre-eclampsia, HELLP syndrome, and eclampsia. similar in 2019 and 2020. The presence of maternal medical conditions documented at delivery increased across conditions studied from 2019 to 2020 . The increase in diabetes appeared to be driven by an increase in gestational diabetes from $8.4 \%$ in 2019 to $9.8 \%$ in 2020 .

Slightly fewer deliveries resulted in ICU admission in 2020 compared to 2019 (1.5\% vs $1.7 \%$, respectively, aPR $=0.90, \mathrm{Cl}: 0.87$, 0.92). Deliveries in 2020 were more likely to result in in-hospital death (aPR $=1.68, \mathrm{Cl}: 1.09,2.59)$, though absolute risk of death was based on few events (2019: $n=33,5$ per 100,000; 2020: $n=53$, 9 per 100,000) (Table 2). When women with COVID-19 at delivery were removed from the analysis, the aPR for in-hospital death in 2020 compared to 2019 was no longer significant (aPR no covid: $1.25, \mathrm{Cl}$ : $0.78,2.00$ ) (Appendix 5). We did not observe differences in the prevalence of livebirths or stillbirths from 2019 to 2020. The prevalence of preterm birth decreased slightly from 2019 to 2020 (9.2\% vs. $9.1 \%$, aPR $=0.96, \mathrm{Cl}: 0.95,0.97)$. There was no change in the prevalence of cesarean delivery from 2019 to 2020 (30.9\% vs. 31.6\%, $\mathrm{aPR}=1.00, \mathrm{Cl}: 1.00,1.01)$. We observed increases of at least $5 \%$ for cesarean deliveries with PROM (aPR: 1.06, Cl: 1.03, 1.08), prolonged labor (aPR: 1.06, Cl: 1.00, 1.15), and attempted forceps or vacuum (aPR: 1.06, Cl: $0.98,1.15$ ). We additionally observed increases in cesarean deliveries considered high-risk due to maternal factors (aPR: $1.07, \mathrm{Cl}: 1.03,1.12$ ) and conduct of labor (aPR: 1.11, Cl: 1.05, 1.17).

The overall distribution of delivery length of stay differed in 2020 compared to 2019 (Fig. 1, $p<0.0001$ ), with a greater proportion of shorter hospital stays occurring after vaginal and cesarean deliveries in 2020 compared to 2019. Further, among vaginal deliveries, after adjusting for within individual and hospital correlations, as well as maternal and hospital characteristics, delivery length of stays less than two days were more common in 2020 compared to 2019 (Table 3). Similarly, among cesarean deliveries, delivery length of stays less than three days were more common in 2020 compared to 2019.

Most results did not substantially differ after excluding women with a COVID-19 diagnosis at delivery $(n=11095$ [1.8\%] of 2020 deliveries) (Appendix 5). When we stratified the analysis by quarter, the distribution of maternal and hospital characteristics was similar to those observed in the main analysis (Appendix 6). Overall, the adverse maternal and pregnancy outcomes were similar by quarter, with overlapping 95\% Cls (Fig. 2; Appendix 7). The aPR for ICU admission and preterm birth was lowest in the second and third quarters, but became null by the fourth quarter. Similarly, in the second quarter, the adjusted prevalence of readmission within 30 days of delivery was approximately $15 \%$ lower in 2020 compared to $2019\left(\mathrm{aPR}_{\mathrm{O} 2}=0.86,95 \% \mathrm{Cl}: 0.81,0.91\right)$; however, the same association was approximately null and no longer significant in the latter two quarters of 2020 (Fig. 2; Appendix 7). The association for stillbirth was increased in the last quarter of $2020\left(\mathrm{aPR}_{\mathrm{Q} 2}=1.08,95 \%\right.$ $\mathrm{Cl}: 1.00,1.17$ ), although the overall prevalence was similar (Fig. 2; Appendix 7). Quarterly associations between delivery length of stay in 2020 compared to 2019 were similar to the main analysis (Appendix 8).

\section{DISCUSSION}

In this analysis comparing deliveries occurring during April through December of 2019 and 2020, we observed statistical differences in delivery outcomes or mode of delivery and complications, but it is unclear whether many of these differences are clinically relevant. The prevalence of preterm birth decreased slightly in the last three quarters of 2020 compared to 2019. The overall change in prevalence was less than $5 \%$, but the decrease is consistent with national estimates [41]. Similarly, cesarean deliveries with PROM, prolonged labor, and attempted forceps or vacuum increased in 2020 compared to 2019, with small absolute and relative differences. Cesarean deliveries considered high-risk due to maternal conditions 
Table 2. Associations between adverse pregnancy outcomes among pregnancies being delivered in April-December 2020 compared to AprilDecember 2019, Premier Healthcare Database Special COVID-19 Release, United States.

\begin{tabular}{|c|c|c|c|c|c|c|}
\hline \multirow[t]{2}{*}{ Total } & \multicolumn{2}{|l|}{2019} & \multicolumn{4}{|l|}{2020} \\
\hline & \multicolumn{2}{|l|}{663,620} & \multicolumn{3}{|l|}{614,093} & \\
\hline \multicolumn{7}{|l|}{ Adverse maternal outcomes } \\
\hline In hospital death & 33 & 5 per 100,000 & 53 & 9 per 100,000 & $1.74(1.12,2.68)$ & $1.68(1.09,2.59)^{b}$ \\
\hline \multicolumn{7}{|l|}{ Pregnancy outcomes } \\
\hline Preterm birth & 61,101 & 9.2 & 56,158 & 9.1 & $0.99(0.98,1.00)$ & $0.96(0.95,0.97)^{c}$ \\
\hline \multicolumn{7}{|l|}{ Mode of delivery } \\
\hline Vaginal & 458,464 & 69.1 & 419,869 & 68.4 & $0.99(0.98,0.99)$ & $1.00(1.00,1.00)^{c}$ \\
\hline Cesarean & 205,156 & 30.9 & 194,224 & 31.6 & $1.03(1.03,1.04)$ & $1.01(1.00,1.01)^{c}$ \\
\hline \multicolumn{7}{|l|}{ Cesarean delivery characteristics } \\
\hline With PROM & 15,231 & 7.4 & 15,083 & 7.8 & $1.05(1.02,1.07)$ & $1.06(1.04,1.08)^{c}$ \\
\hline \multicolumn{7}{|l|}{ SMFM cesarean delivery designation } \\
\hline Low-risk $^{d}$ & 65,952 & 32.2 & 64,369 & 33.1 & $1.03(1.02,1.04)$ & $1.04(1.03,1.04)^{c}$ \\
\hline High-risk $^{e}$ & 139,204 & 67.9 & 129,855 & 66.9 & $0.98(0.98,0.99)$ & $0.98(0.98,0.99)^{c}$ \\
\hline Due to maternal factors & 4609 & 2.3 & 4838 & 2.5 & $1.11(1.06,1.15)$ & $1.07(1.03,1.12)^{c}$ \\
\hline Due to preterm birth & 26,944 & 13.1 & 25,667 & 13.2 & $1.01(0.99,1.02)$ & $0.98(0.96,0.99)^{c}$ \\
\hline Due to stillborn & 727 & 0.4 & 719 & 0.4 & $1.03(0.93,1.15)$ & $1.02(0.92,1.14)^{c}$ \\
\hline Due to malpresentation & 26,555 & 12.9 & 25,204 & 13.0 & $1.00(0.99,1.02)$ & $1.01(0.99,1.02)^{c}$ \\
\hline Due to fetal factors & 234 & 0.1 & 224 & 0.1 & $1.01(0.84,1.21)$ & $1.00(0.83,1.20)^{c}$ \\
\hline Due to uterine/placental factors & 105,848 & 51.6 & 97,937 & 50.4 & $0.97(0.97,0.98)$ & $0.97(0.97,0.98)^{c}$ \\
\hline Due to conduct of labor & 2553 & 1.2 & 2642 & 1.4 & $1.09(1.04,1.15)$ & $1.11(1.05,1.17)^{c}$ \\
\hline Readmission within 30 days of delivery & 8224 & 1.2 & 7665 & 1.3 & $1.01(0.98,1.04)$ & $0.97(0.94,1.00)^{c}$ \\
\hline
\end{tabular}

Abbreviations: Cl confidence interval, PROM Premature rupture of membranes, SMFM Society for Maternal-Fetal Medicine.

${ }^{a}$ Model adjusted for individual women nested within hospitals (to account for within person and facility correlation), continuous maternal age, payor, hypertensive disorders, diabetes, and obesity.

${ }^{\mathrm{b}}$ Model adjusted for the hospital (to account for within facility correlation), categorized maternal age (15-19, 20-24, 25-29, 30-34, 35-39, 40-45), payor, hypertensive disorders, diabetes, and obesity.

'Models adjusted for the hospital (to account for within facility correlation), continuous maternal age, division, payor, hypertensive disorders, diabetes, and obesity.

${ }^{\mathrm{d}} \mathrm{A}$ cesarean delivery without any high-risk factors was considered a delivery at low-risk of cesarean delivery.

${ }^{\mathrm{e}} \mathrm{A}$ delivery with medical factors making it high-risk for cesarean delivery; factors are not mutually exclusive.

and conduct of labor increased in 2020 compared to 2019. Among all pregnancies, delivery length of stay was shorter for deliveries in 2020 compared to 2019, likely due to COVID-19 mitigation measures. Among all women included in our sample, in-hospital death increased 69\% from 2019 to 2020, although the absolute risk was less than $0.01 \%$ in both years. However, in the sensitivity analysis that excluded women with a COVID-19 diagnosis at delivery from the 2020 sample, the association was no longer significant. These results support the hypothesis that COVID-19 likely drove the increase in in-hospital deaths, but further exploration of this outcome, with in-depth reviews from maternal mortality review committees, is needed to elucidate the cause of deaths. The sensitivity analysis assessing differences by quarter suggests that overall, outcomes were stable throughout 2020; the exceptions were preterm birth, which decreased early in the pandemic but became null in the latter half of the year, maternal ICU admission, which followed a similar pattern, and stillbirth, which increased in the last quarter of 2020.

Our estimates of changes in pregnancy outcomes and mode of delivery add to the literature on the impact of the COVID-19 pandemic on pregnancy outcomes. While some U.S. and
European studies have observed reductions in preterm birth $[22-24,26,29,32,34]$, others have not $[28,30,31,33]$. Our data suggest that, after adjustment, pregnancies delivering in the last three quarters of 2020 were slightly less likely to deliver preterm compared to those delivering in 2019. The reduction in the absolute prevalence of preterm birth from 2019 to 2020 was small. However, this change in prevalence represents approximately 382 fewer preterm births occurring in 2020 than would be expected using the prevalence from 2019. Infants born preterm can experience long-term health problems, and the costs of caring for children born preterm in the United States are substantial [42]. Even modest reductions in preterm birth may have important public health implications. Some studies have observed increases in stillbirth rates during the pandemic [14, 15]. However, other evidence suggests that stillbirth may not be increased during the pandemic period [26, 31, 43]. Our data do not suggest an increase in stillbirths between April-December 2020 , although we did observe a slight increase in stillbirth in the last quarter of 2020 compared to 2019. The mechanism driving changes in pregnancy and delivery outcomes during the pandemic is unclear. Strict mitigation measures, implemented 
a.Length of delivery hospitalization stay among women with vaginal deliveries, 2019

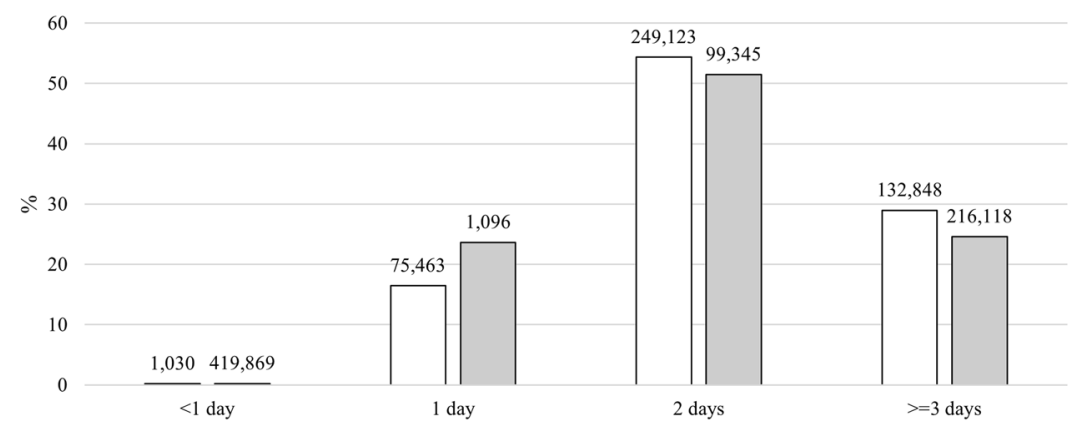

b. Length of delivery hospitalization stay among women with cesarean deliveries, 2019 $(n=205,159)$ and $2020(n=194,224)$



Fig. 1 Length of delivery hospitalization stay among women by mode of delivery, April-December 2019 and 2020, Premier Healthcare Database Special COVID-19 Release, United States. Numbers above bars represent counts. a Represents length of delivery hospitalization stay among vaginal deliveries; $\mathbf{b}$ represents length of delivery hospitalization stay among cesarean deliveries.

Table 3. Association between delivery length of stay among pregnancies being delivered in April-December 2020 compared to April-December 2019, Premier Healthcare Database Special COVID-19 Release, United States.

\begin{tabular}{|c|c|c|}
\hline & Prevalence ratio $^{a}$ & Adjusted prevalence ratio ${ }^{a, b}$ \\
\hline \multicolumn{3}{|c|}{ Vaginal deliveries } \\
\hline 2 days $^{c}$ & 1.00 & 1.00 \\
\hline$<1$ day & $1.23(1.13,1.34)$ & $1.32(1.21,1.44)$ \\
\hline 1 day & $1.36(1.35,1.37)$ & $1.39(1.38,1.40)$ \\
\hline$\geq 3$ days & $0.93(0.92-0.94)$ & $0.90(0.90,0.91)$ \\
\hline \multicolumn{3}{|c|}{ Cesarean deliveries } \\
\hline 3 days $^{c}$ & 1.00 & 1.00 \\
\hline$<1$ day & $1.38(1.27,1.50)$ & $1.53(1.40,1.66)$ \\
\hline $1-2$ days & $1.30(1.29,1.31)$ & $1.32(1.31,1.32)$ \\
\hline 4 days & $0.99(0.98,1.00)$ & $0.96(0.95,0.98)$ \\
\hline$\geq 5$ days & $1.01(1.00,1.03)$ & $0.94(0.92,0.95)$ \\
\hline
\end{tabular}

aseparate models were run comparing 2020 deliveries to 2019 deliveries for each length of stay category, compared to the reference category.

${ }^{\mathrm{b}}$ Model adjusted for individual women nested within the hospital (to account for within person and facility correlation), census division, continuous maternal age, payor, hypertensive disorders, diabetes, and obesity.

'Reference category.

early in the pandemic, such as stay at home orders, however, may play a role, and may also explain why the greatest reduction in preterm birth was observed early in the pandemic [44].

Our assessment of mode of delivery and characteristics of cesarean deliveries provides evidence that the COVID-19 pandemic has not substantially changed cesarean delivery practices in the hospitals included in this analysis, in accordance with ACOG recommendations [5]. The lack of difference between the prevalence of cesarean deliveries in 2020 compared to 2019 is consistent with what has been observed within the U.S. and other countries [26, 41, 43, 45, 46]. Further, the shortened delivery hospitalization lengths of stay are also consistent with the literature and ACOG recommendations $[5,47]$. The impact of shortened delivery hospitalizations on postpartum health remains to be studied; in our analytic sample, the prevalence of maternal hospital readmission within 30 days slightly decreased from 2019 to 2020.

The findings of this analysis should be interpreted considering several limitations. First, we used ICD-10-CM codes in administrative discharge data to identify delivery, pregnancy, and maternal outcomes, which may have resulted in misclassification of outcomes, including COVID-19 diagnosis. ICD-10-CM codes do not provide information on COVID-19 symptom severity. Second, among women with multiple delivery hospitalizations separated by 30 days or less, the first delivery hospitalization encounter was used to determine adverse maternal and pregnancy outcomes; this could have resulted in misclassification of outcomes if the subsequent hospitalizations differed in recorded diagnoses. Third, because our analysis was limited to delivery hospitalizations and linkage across hospitals is not available, we did not have information on maternal health during pregnancy, including COVID-19 diagnosis during pregnancy, maternal pregnancy history (including prior cesarean delivery), maternal use of prenatal care, or hospitalizations occurring outside of the delivery hospital after pregnancy. We were also unable to link maternal and infant outcomes; thus, this analysis is focused only on selected maternal, delivery, and pregnancy outcomes present at the 


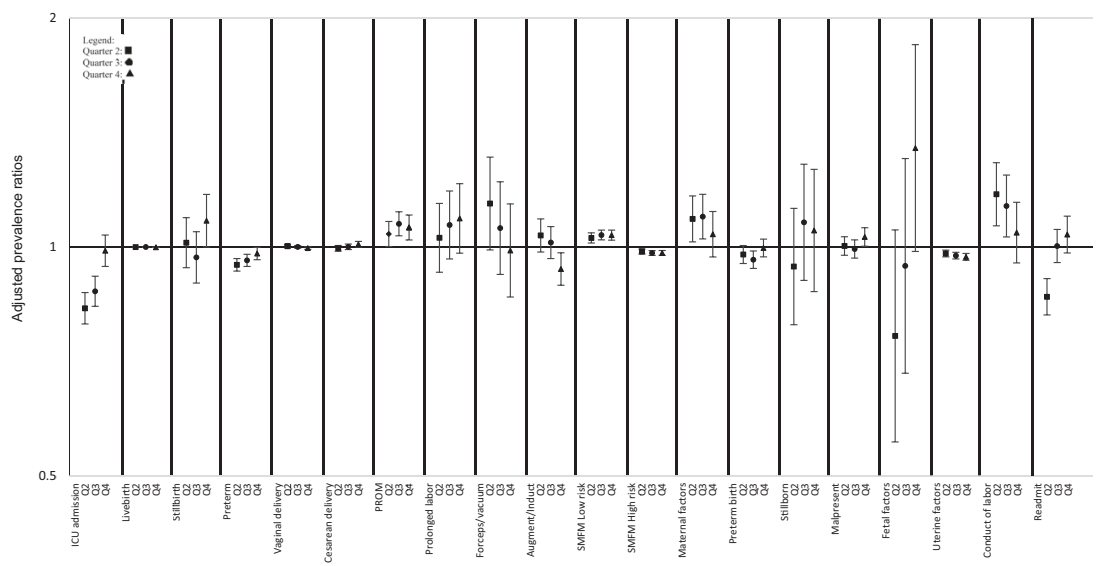

Fig. 2 Association between adverse pregnancy outcomes among pregnancies being delivered in April-December 2020 compared to April-December 2019, stratified by quarter, Premier Healthcare Database Special COVID-19 Release, United States. Maternal ICU admission adjusted for individual women nested within hospitals (to account for within person and facility correlation), continuous maternal age, payor, hypertensive disorders, diabetes, and obesity; all other models adjusted for hospital (to account for within facility correlation), continuous maternal age, division, payor, hypertensive disorders, diabetes, and obesity. PROM, prolonged labor, forceps/vacuum, augment/ induct are cesarean delivery characteristics; Maternal factors, preterm birth stillborn, uterine factors, conduct of labor are SMFM high-risk characteristics. Quarter 2 represented deliveries occurring April-June; Quarter 3 represented deliveries occurring July-September; Quarter 4 represented deliveries occurring October-December. Abbreviations: Augment/induct augmentation or induction of labor; ICU intensive care unit, Malpresent malpresenation, PROM premature rupture of membrane, Q2 quarter 2, Q3 quarter 3, Q4 quarter 4, Readmit 30-day readmission, SMFM society for maternal fetal medicine, Uterine factors uterine/placental factors.

delivery hospitalization. Fourth, although we have a large population of delivery hospitalizations across the United States, the PHD-SR data are not nationally representative. Fifth, although the PHD-SR data include information on potential confounders, unmeasured confounding may exist. Finally, as the data included in PHD-SR only included hospitalizations from 2019 on, this analysis only included one year of baseline data to compare to outcomes in 2020, which could impact our results. It is possible that analyses incorporating additional years of data may yield different results. Further, as the COVID-19 pandemic has continued beyond 2020, its impact on pregnancy and delivery outcomes may continue to evolve.

Despite these limitations, our analysis had several strengths. The PHD-SR data are geographically diverse, allowing us to include hospitalizations from across the United States. Additionally, our population is racially diverse and represents deliveries occurring among multiple payor types. The large sample size allowed investigation of multiple pregnancy outcomes, mode of delivery and complications, and maternal outcomes that have not been well studied during the COVID-19 pandemic.

During the first nine months of the COVID-19 pandemic, fewer deliveries were preterm compared to women delivering during the same period in 2019. However, this reduction was only observed early in the pandemic. Overall, no changes in stillbirth were observed, although the quarterly analysis yielded slightly different patterns. The proportion of cesarean deliveries with PROM, prolonged labor, and attempted forceps or vacuum increased, as did cesarean deliveries with selected high-risk characteristics. We observed an increase in inhospital death in 2020 compared to 2019, which should be further investigated. Of note, excluding women with COVID-19 diagnosis at delivery resulted in a difference that was not statistically significant. Future research should focus on identifying factors (e.g., COVID-19 mitigation measures, changes in clinical practices, maternal stress, etc.) that contributed to these changes in pregnancy outcomes.

\section{DISCLAIMER}

The findings and conclusions in this report are those of the authors and do not necessarily represent the views of the Centers for Disease Control and Prevention.

\section{DATA AVAILABILITY}

The authors cannot make data and study materials available to other investigators for purposes of reproducing the results because of licensing restrictions. Interested parties, however, could obtain and license the data by contacting Premier.

\section{REFERENCES}

1. Whaley CM, Pera MF, Cantor J, Chang J, Velasco J, Hagg HK, et al. Changes in health services use among commercially insured US populations during the COVID-19 pandemic. JAMA Netw Open. 2020;3:e2024984.

2. Hartnett KP, Kite-Powell A, DeVies J, Coletta MA, Boehmer TK, Adjemian J, et al. Impact of the COVID-19 pandemic on emergency department visits-United States, January 1, 2019-May 30, 2020. MMWR Morb Mortal Wkly Rep. 2020;69:699-704.

3. Fryer K, Delgado A, Foti T, Reid CN, Marshall J. Implementation of obstetric telehealth during COVID-19 and beyond. Matern Child Health J. 2020;24:1104-10.

4. Society for Maternal-Fetal Medicine. The Society for Maternal-Fetal Medicine COVID19 ultrasound clinical practice suggestions 2020 updated October 20, 2020. https://s3. amazonaws.com/cdn.smfm.org/media/2550/Ultrasound_Covid19_Suggestions_ 10-20-20_(final).pdf.

5. The American College of Obstetricians and Gynecologists. COVID-19 FAQs for obstetricians-gynecologists, obstetrics Washington, DC2020. https://www.acog. org/clinical-information/physician-faqs/covid-19-faqs-for-ob-gyns-obstetrics.

6. Centers for Disease Control and Prevention. Considerations for inpatient obstetric healthcare settings 2020. https://www.cdc.gov/coronavirus/2019-ncov/hcp/ inpatient-obstetric-healthcare-guidance.html.

7. Tull MT, Edmonds KA, Scamaldo KM, Richmond JR, Rose JP, Gratz KL. Psychological outcomes associated with stay-at-home orders and the perceived impact of COVID-19 on daily life. Psychiatry Res. 2020;289:113098.

8. Flanagan EW, Beyl RA, Fearnbach SN, Altazan AD, Martin CK, Redman LM. The impact of COVID-19 stay-at-home orders on health behaviors in adults. Obesity. 2021;29:438-45.

9. Moreland A, Herlihy C, Tynan MA, Sunshine G, McCord RF, Hilton C, et al. Timing of state and territorial COVID-19 stay-at-home orders and changes in population movement-United States, March 1-May 31, 2020. MMWR Morb Mortal Wkly Rep. 2020;69:1198-203.

10. Marroquin B, Vine V, Morgan R. Mental health during the COVID-19 pandemic: effects of stay-at-home policies, social distancing behavior, and social resources. Psychiatry Res. 2020;293:113419.

11. Delahoy MJ, Whitaker M, O'Halloran A, Chai SJ, Kirley PD, Alden N, et al. Characteristics and maternal and birth outcomes of hospitalized pregnant women with laboratory-confirmed COVID-19 - COVID-NET, 13 States, March 1-August 22, 2020. MMWR Morb Mortal Wkly Rep. 2020;69:1347-54.

12. Ko JY, DeSisto CL, Simeone RM, Ellington S, Galang RR, Oduyebo T, et al. Adverse pregnancy outcomes, maternal complications, and severe illness among U.S. 
delivery hospitalizations with and without a COVID-19 diagnosis. Clin Infect Dis. 2021;73(Suppl 1):S24-31.

13. Zambrano LD, Ellington S, Strid P, Galang RR, Oduyebo T, Tong VT, et al. Update: Characteristics of symptomatic women of reproductive age with laboratoryconfirmed SARS-CoV-2 infection by pregnancy status-United States, January 22October 3, 2020. MMWR Morb Mortal Wkly Rep. 2020;69:1641-7.

14. Khalil A, von Dadelszen P, Draycott T, Ugwumadu A, O'Brien P, Magee L. Change in the incidence of stillbirth and preterm delivery during the COVID-19 pandemic. JAMA. 2020;324:705-6.

15. De Curtis M, Villani L, Polo A. Increase of stillbirth and decrease of late preterm infants during the COVID-19 pandemic lockdown. Arch Dis Child Fetal Neonatal Ed. 2020;106:456.

16. Karasek D, Baer RJ, McLemore MR, Bell AJ, Blebu BE, Casey JA, et al. The association of COVID-19 infection in pregnancy with preterm birth: a retrospective cohort study in California. Lancet Reg Health Am. 2021;2:100027.

17. DeSisto CL, Wallace B, Simeone RM, Polen K, Ko JY, Meaney-Delman D, et al. Risk for stillbirth among women with and without COVID-19 at delivery hospitalization-United States, March 2020-September 2021. MMWR Morb Mortal Wkly Rep. 2021;70:1640-5.

18. Allotey J, Stallings E, Bonet M, Yap M, Chatterjee S, Kew T, et al. Clinical manifestations, risk factors, and maternal and perinatal outcomes of coronavirus disease 2019 in pregnancy: living systematic review and meta-analysis. BMJ. 2020;370:m3320.

19. Chmielewska B, Barratt I, Townsend R, Kalafat E, van der Meulen J, Gurol-Urganci I, et al. Effects of the COVID-19 pandemic on maternal and perinatal outcomes: a systematic review and meta-analysis. Lancet. Glob Health. 2021;9:e759-e72.

20. Burki T. The indirect impact of COVID-19 on women. Lancet Infect Dis. 2020;20:904-5.

21. Research Committee, Society for Maternal-Fetal Medicine (SMFM), Boelig RC, Aagaard KM, Debbink MP, Shamshirsaz AA, SMFM Research Committee. Society for Maternal-Fetal Medicine Special Statement: COVID-19 research in pregnancy: progress and potential. Am J Obstet Gynecol. 2021;225:B19-B31.

22. Hedermann G, Hedley PL, Baekvad-Hansen M, Hjalgrim H, Rostgaard K, Poorisrisak $P$, et al. Danish premature birth rates during the COVID-19 lockdown. Arch Dis Child Fetal Neonatal Ed. 2021;106:93-5.

23. Been JV, Burgos Ochoa L, Bertens LCM, Schoenmakers S, Steegers EAP, Reiss IKM. Impact of COVID-19 mitigation measures on the incidence of preterm birth: a national quasi-experimental study. Lancet Public Health. 2020;5:e604-e11.

24. Matheson A, McGannon CJ, Malhotra A, Palmer KR, Stewart AE, Wallace EM, et al. Prematurity rates during the Coronavirus Disease 2019 (COVID-19) Pandemic lockdown in Melbourne, Australia. Obstet Gynecol. 2021;137:405-7.

25. Philip RK, Purtill H, Reidy E, Daly M, Imcha M, McGrath D, et al. Unprecedented reduction in births of very low birthweight (VLBW) and extremely low birthweight (ELBW) infants during the COVID-19 lockdown in Ireland: a 'natural experiment' allowing analysis of data from the prior two decades. BMJ Glob Health. 2020;5:e003075.

26. McDonnell S, McNamee E, Lindow SW, O'Connell MP. The impact of the Covid-19 pandemic on maternity services: A review of maternal and neonatal outcomes before, during and after the pandemic. Eur J Obstet Gynecol Reprod Biol. 2020:255:172-6.

27. Sun S, Savitz DA, Wellenius GA. Changes in adverse pregnancy outcomes associated with the COVID-19 Pandemic in the United States. JAMA Netw Open. 2021;4:e2129560.

28. Son M, Gallagher K, Lo JY, Lindgren E, Burris HH, Dysart K, et al. Coronavirus disease 2019 (COVID-19) pandemic and pregnancy outcomes in a U.S. population. Obstet Gynecol. 2021;138:542-51.

29. Harvey EM, McNeer E, McDonald MF, Shapiro-Mendoza CK, Dupont WD, Barfield W, et al. Association of preterm birth rate with COVID-19 statewide stay-at-home orders in Tennessee. JAMA Pediatr. 2021;175:635-37.

30. Main EK, Chang SC, Carpenter AM, Wise PH, Stevenson DK, Shaw GM, et al. Singleton preterm birth rates for racial and ethnic groups during the coronavirus disease 2019 pandemic in California. Am J Obstet Gynecol. 2021;224:239-41.

31. Handley SC, Mullin AM, Elovitz MA, Gerson KD, Montoya-Williams D, Lorch SA, et al. Changes in preterm birth phenotypes and stillbirth at 2 Philadelphia hospitals during the SARS-CoV-2 pandemic, March-June 2020. JAMA. 2021;325:87-9.

32. Berghella V, Boelig R, Roman A, Burd J, Anderson K. Decreased incidence of preterm birth during coronavirus disease 2019 pandemic. Am J Obstet Gynecol MFM. 2020;2:100258.

33. Wood R, Sinnott C, Goldfarb I, Clapp M, McElrath T, Little S. Preterm birth during the coronavirus disease 2019 (COVID-19) pandemic in a large hospital system in the United States. Obstet Gynecol. 2021;137:403-4.

34. Gemmill A, Casey JA, Catalano R, Karasek D, Margerison CE, Bruckner T. Changes in preterm birth and Caesarean deliveries in the United States during the SARSCoV-2 pandemic. Paediatr Perinat Epidemiol. 2021;13:10.1111/ppe.12811. https:// doi.org/10.1111/ppe.12811. Epub ahead of print.
35. Boelig RC, Manuck T, Oliver EA, Di Mascio D, Saccone G, Bellussi F, et al. Labor and delivery guidance for COVID-19. Am J Obstet Gynecol MFM. 2020;2:100110.

36. Society for Maternal-Fetal Medicine and Society for Obstetric and Anesthesia and Perinatology. Labor and delivery COVID-19 considerations 2020. https:// s3.amazonaws.com/cdn.smfm.org/media/2277/SMFM-SOAP_COVID_LD_ Considerations_3-27-20_(final)_PDF.pdf.

37. Premier Healthcare Database white paper: Data that informs and performs: Premier; updated Mar. 2, 2020. http://offers.premierinc.com/rs/381-NBB-525/ images/PHD_COVID-19_White_Paper.pdf.

38. Armstrong J, McDermott P, Saade GR, Srinivas SK, Society for Maternal-Fetal Medicine Health $P$, Advocacy $C$. et al. Coding update of the SMFM definition of low risk for cesarean delivery from ICD-9-CM to ICD-10-CM. Am J Obstet Gynecol. 2017;217:B2-B12. e56

39. Zou G. A modified Poisson regression approach to prospective studies with binary data. Am J Epidemiol. 2004;159:702-6.

40. Centers for Disease Control and Prevention. New ICD-10-CM code for the 2019 novel coronavirus (COVID-19), April, 2020 2020. https://www.cdc.gov/nchs/data/ icd/Announcement-New-ICD-code-for-coronavirus-3-18-2020.pdf.

41. Hamilton B, Martin J, Osterman M. Births: Provisional data for 2020. Vital Statistics Rapid Release: National Center for Health Statistics. May 2021.

42. Frey HA, Klebanoff MA. The epidemiology, etiology, and costs of preterm birth. Semin Fetal Neonatal Med. 2016;21:68-73.

43. Meyer R, Bart Y, Tsur A, Yinon Y, Friedrich L, Maixner N, et al. A marked decrease in preterm deliveries during the coronavirus disease 2019 pandemic. Am J Obstet Gynecol. 2021;224:234-7.

44. Rolnik DL, Matheson A, Liu Y, Chu S, McGannon C, Mulcahy B, et al. The impact of COVID-19 pandemic restrictions on pregnancy duration and outcomes in Melbourne, Australia. Ultrasound Obstet Gynecol. 2021;58:677-87.

45. Greene NH, Kilpatrick SJ, Wong MS, Ozimek JA, Naqvi M. Impact of labor and delivery unit policy modifications on maternal and neonatal outcomes during the coronavirus disease 2019 pandemic. Am J Obstet Gynecol MFM 2020;2:100234.

46. Bhatia K, Columb M, Bewlay A, Eccles J, Hulgur M, Jayan N, et al. The effect of COVID-19 on general anaesthesia rates for caesarean section. A cross-sectional analysis of six hospitals in the north-west of England. Anaesthesia. 2021;76:312-9.

47. Bornstein E, Gulersen M, Husk G, Grunebaum A, Blitz MJ, Rafael TJ, et al. Early postpartum discharge during the COVID-19 pandemic. J Perinat Med. 2020;48:1008-12

\section{ACKNOWLEDGEMENTS}

John House, Premier Inc. for collecting, managing, and granting access to the dataset CDC COVID-19 Response Data, Analytics and Visualization Task Force for data acquisition and analytic guidance; CDC COVID-19 Response Epidemiology Task Force for scientific guidance. No funding was obtained for this work.

\section{AUTHOR CONTRIBUTIONS}

All authors contributed to the conceptualization, data interpretation, and manuscript review and editing. $\mathrm{BW}, \mathrm{CD}, \mathrm{KD}$, and $\mathrm{RS}$ were involved with conceptualization, formal analysis, data interpretation, methodology, validation, and writing-review and editing. RS additionally contributed writing - original draft. JK, LZ, RG, SE, and VT were involved with conceptualization, methodology, data interpretation, and writing-review and editing.

\section{COMPETING INTERESTS}

The authors declare no competing interests.

\section{ADDITIONAL INFORMATION}

Supplementary information The online version contains supplementary material available at https://doi.org/10.1038/s41372-022-01327-3.

Correspondence and requests for materials should be addressed to Regina $M$. Simeone.

Reprints and permission information is available at http://www.nature.com/ reprints

Publisher's note Springer Nature remains neutral with regard to jurisdictional claims in published maps and institutional affiliations. 